\title{
Caracterización de la harina de quinua (Chenopodium quinoa Willd.) y la harina de tarwi (Lupinus mutabilis Sweet) para su industrialización
}

\section{Characterization of quinoa flour (Chenopodium quinoa Willd.) and tarwi flour (Lupinus mutabilis Sweet) for industrialization}

\author{
(iD) 'Lucia Pantoja-Tirado (iD ' 'Gino Prieto-Rosales (iD ${ }^{2}$ Elza Aguirre Vargas \\ Universidad Nacional Autónoma de Tayacaja "Daniel Hernández Morillo". Pampas, Perú. \\ Universidad Nacional del Santa. Santa, Perú.
}

\section{Resumen}

La harina de granos andinos son una fuente rica en proteínas y carbohidratos es por ello la importancia de ser caracterizados, algunos de estos granos andinos son la quinua (Chenopodium quinoa Willd.) y el tarwi (Lupinus mutabilis Sweet). El presente trabajo de investigación tiene como objetivo realizar la caracterización de la harina de quinua (Chenopodium quinoa Willd.) y de la harina de tarwi (Lupinus mutabilis Sweet) para su industrialización. Se determinó que la composición química proximal de la harina de quinua y es como sigue: Proteína (9.05\%), Humedad (10.48 $\pm 0.12 \%)$, Cenizas (2.44 $\pm 0.05 \%)$, Grasa (2.53 $\pm 0.02 \%)$, Fibra $(3.11 \pm 0.03 \%)$ y Carbohidratos $(72.39 \%)$ y también la composición química proximal de la harina de tarwi y es como sigue: Proteína (49.04\%), Humedad $(6.33 \pm 0.15 \%)$, Cenizas (2.85 $\pm 0.04 \%)$, Grasa (27.32 $\pm 0.25 \%)$, Fibra $(2.94 \pm 0.05 \%)$ y Carbohidratos (11.52\%). Se evidencia que estas harinas tienen un alto contenido de proteína y carbohidratos, y pueden ser considerados como materias primas potenciales para la elaboración de alimentos nutritivos.

Palabras clave: Chenopodium quinoa Willd; Lupinus mutabilis Sweet; Harina de quinua; Harina de tarwi; industrialización.

Recibido: Marzo 2020

Aceptado: Junio 2020

\section{Abstract}

Andean grain flour is a rich source of protein and carbohydrates, which is why it is important to be characterized, some of these Andean grains are quinoa (Chenopodium quinoa Willd.) And tarwi (Lupinus mutabilis Sweet). The objective of this research work is to characterize quinoa flour (Chenopodium quinoa Willd.) And tarwi flour (Lupinus mutabilis Sweet) for its industrialization. It was determined that the chemical composition of quinoa flour is as follows: Protein (9.05\%), Moisture (10.48 $\pm 0.12 \%)$, Ash (2.44 $\pm 0.05 \%)$, Fat (2.53 $\pm 0.02 \%)$, Fiber (3.11 $\pm 0.03 \%)$ and Carbohydrates (72.39\%) and also the proximal chemical composition of tarwi flour and is as follows: Protein (49.04\%), Humidity (6.33 \pm $0.15 \%)$, Ashes (2.85 $\pm 0.04 \%)$, Fat ( $27.32 \pm 0.25 \%)$, Fiber ( $2.94 \pm 0.05 \%)$ and Carbs $(11.52 \%)$. It is evident that these flours have a high protein and carbohydrate 
content, and can be considered as potential raw materials for the production of nutritious foods.

Keywords: Chenopodium quinoa Willd; Lupinus mutabilis Sweet; Quinoa flour; Tarwi flour; industrialization.

\section{INTRODUCCIÓN}

Los problemas de malnutrición y hambre en los países en desarrollo, se sustentan en la falta de inclusión de micronutrientes en la dieta y en el bajo consumo de alimentos que contengan buena disponibilidad de proteína, energía y micronutrientes; afirmaciones que son corroboradas por el Instituto Nacional de Estadística e Informática (INEI, 2018).

La harina de quinua el cual tiene un excepcional valor nutritivo, con grandes cantidades de carbohidratos, proteínas vegetales y un excelente balance de aminoácidos esenciales y fibra, es el cereal de mayor y más completa composición en aminoácidos que existe en nuestro planeta ya que contiene aminoácidos esenciales como la luteína y la lisina esenciales para el ser humano (Murphy y Matanguihan, 2015). La harina de tarwi tiene un alto valor nutritivo, que se distingue por su alto contenido de proteína (Navia-Coarite et al., 2019). Debido a su alto contenido de proteína y grasas, el tarwi (Lupinus mutabilis Sweet) es un cultivo de los andes del Perú, Ecuador y Bolivia en donde se consume por su alto valor proteico, fibra y contenido de grasas (Granados et al., 2019). El incremento del consumo de tarwi mejorará la salud y el estado nutricional de las personas.

En objetivo de esta investigación fue evaluar la composición centesimal de la harina de quinua (Chenopodium quinoa Willd.) variedad Blanca de Juli y la harina de tarwi (Lupinus mutabilis Sweet) variedad Puno, para su industrialización.

\section{MATERIALES Y MÉTODOS}

La investigación se realizó en los siguientes ambientes: instituto de investigación tecnológico agroindustrial, laboratorio de investigación y desarrollo de productos agroindustriales y el laboratorio de análisis y composición de produc- tos agroindustriales de la Universidad Nacional del Santa.

La quinua (Chenopodium quinoa Willd) en grano de la variedad Blanca de Juli y el tarwi (Lupinus mutabilis Sweet) en grano de la variedad Puno; se adquirió del fundo de la familia Pantoja, localizada en las coordenadas UTM Longitud: 827802.33m y Latitud: $9084090.72 m$. La harina de quinua fue obtenida siguiendo la metodología de Calla \& Cortez (2011) y la harina de tarwi siguiendo la metodología de Salcedo (2009).

Equipos: Mufla, THERMOLYNE; pH metro digital, HACH; Balanza Analítica, PRECISA GRAVIMETRICS A G.; Estufa, POL-EKO APARATURA; Equipo de Actividad de Agua, ROTRONIC; Colorímetro, KONIKA MINOLTA; Soxhlet, FOSS; Secadora de Bandejas, JARCON DEL PERÚ; LabQuest vernier; Sensor de Humedad Relativa (código RH-BTA); Módulo de Molienda y Tamizado, JARCON DEL PERÚ; Cocina a gas, FADIC; Autoclave, SELECTA y Refrigeradora, $\mathrm{BOSCH}$.

Materiales de vidrio: probetas $(50,100$ y $500 \mathrm{ml})$, varilla de vidrio, vasos precipitados (500 y $1000 \mathrm{ml})$, matraces erlenmeyer (250 y $500 \mathrm{ml})$, pipetas (1, 5 y $10 \mathrm{ml})$, placas petri.

Reactivos: fenolftaleína, agua destilada, solución de hidróxido de sodio (0.1n y 0.255n), ácido sulfúrico a $0.255 n$, hexano a $98 \%$, cloroformo, ácido acético glacial, solución saturada de ioduro de potasio, solución $0.1 \mathrm{n}$ de tiosulfato de sodio, almidón al 1\%.

\section{Obtención de la harina de quinua}

La obtención de la harina de quinua se basó en la metodología de Calla \& Cortez (2011). El flujo para la elaboración de la harina de 
quinua se presenta en la figura 1, cuya descripción se presenta a continuación:

Eliminación de impurezas: los granos de quinua fueron seleccionados de manualmente, con el objetivo de separar los granos dañados o contaminados, piedras, trozos de metales, etc.

Lavado del grano: el grano se lavó con agua a temperatura ambiente aproximadamente unos 40 minutos. Se lavó sucesivas veces una cierta cantidad de granos (aprox. $1 / 2 \mathrm{~kg}$ ) con agua, en la proporción Quinua:Agua/1:2; has- ta que no produzca espuma. Se desinfecto el grano con hipoclorito de sodio a una concentración de 10 ppm durante 5 minutos.

Secado: el secado se realizó aproximadamente por 4.5 horas a una temperatura de $60^{\circ} \mathrm{C}$ hasta alcanzar una humedad del 12\%; en un secador de bandejas de marca JARCON.

Molienda y tamizado: con esta operación se uniformiza las partículas de quinua, obteniéndose harina de quinua. Se realizó en el módulo de molienda y tamizado de marca JARCON; para el tamizado se utilizó el tamiz de número 120.

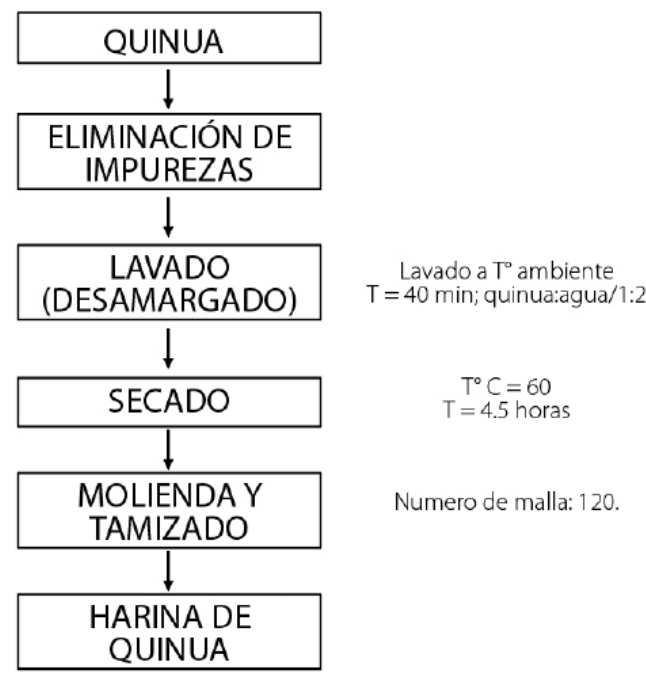

Figura 1. Flujo de operaciones para la obtención de harina de quinua.

\section{Obtención de la harina de tarwi}

La obtención de la harina de tarwi se basó en la metodología de Salcedo (2009). El flujo para la elaboración de la harina de tarwi se presenta en la figura 2, cuya descripción se presenta a continuación:

Limpieza y selección: los granos de tarwi fueron seleccionados de manualmente, con el objetivo de separar los granos dañados o contaminados, basura, piedras, metales, etc.

Hidratación: se hidrato los granos de tarwi seleccionados, remojándolos en agua, en la proporción Tarwi:Agua / 1:4, durante 12 horas (según el método recomendado por Salcedo, 2009), para ablandar los granos y hacer menos drástica la cocción.

Pre-Cocción: se hirvió el tarwi remojado por un tiempo de 5 minutos, luego, se dejó reposar durante 30 minutos en la misma agua caliente. Esto se realiza para ablandar la cascara del grano y facilitar el pelado.

Pelado: luego se enfrió el tarwi cocinado, agregándole agua fría hasta llegar a $35-40^{\circ} \mathrm{C}$. Se eliminó la cascara manualmente, para facilitar las etapas de cocción, secado del grano y a molienda. La cascara se elimina friccionando el grano cocido. 
Cocción: se realizó por un tiempo de 40minutos, con dos cambios de agua cada 20 minutos, contados desde el momento que inicia a hervir, a la temperatura de $96-98^{\circ} \mathrm{C}$, para eliminar gran parte de los alcaloides en el agua.

Lavado con agua: sirve para eliminar los alcaloides que contiene el grano de tarwi, reduciéndole hasta un máximo de 0.02\%. En esta etapa se realizó varios cambios sucesivos de agua cada 8 horas por espacio de 3.5 dias, hasta que el agua resulte cristalina y sin sabor amargo. Se desinfecto el grano con hipoclorito de sodio a una concentración de 10 ppm durante 5 minutos.

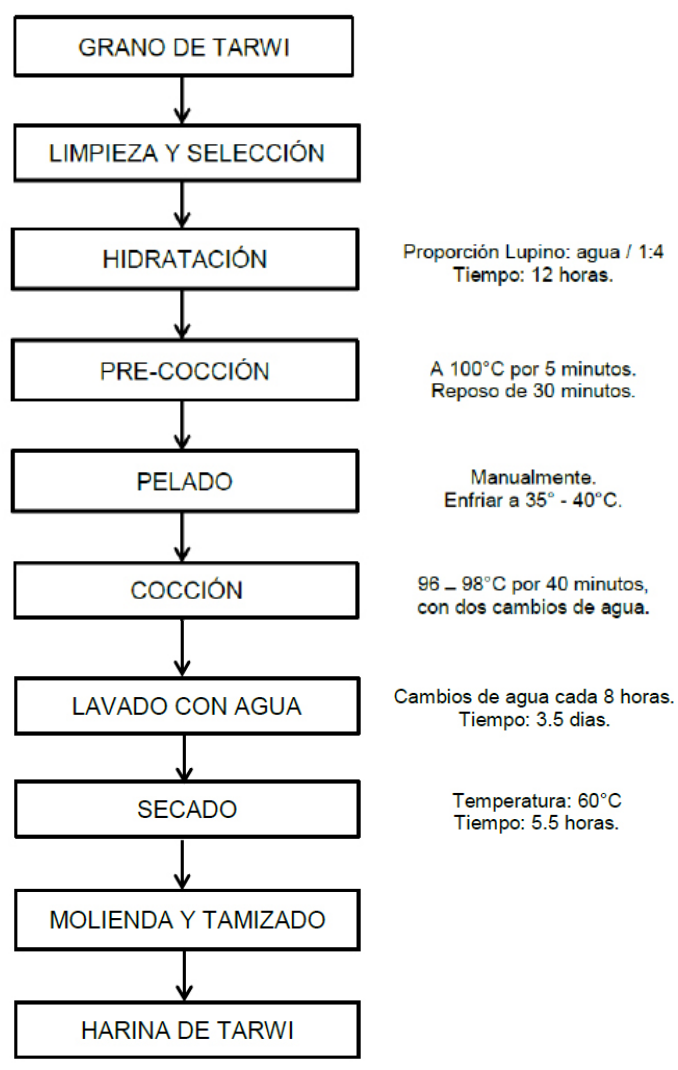

Figura 2. Flujo de operaciones para la obtención de harina de tarwi.

\section{Caracterización de las harinas de quinua y tarwi:}

La caracterización se realizó individualmente a la harina de quinua y a la harina de tarwi. Los análisis se realizaron en el Instituto de Investigación Tecnológica Agroindus-
Secado: con el secado se redujo la disponibilidad de agua en el grano. Se realizó en el secador de bandejas de marca JARCON. El secado duro en promedio 5.5 horas y la temperatura promedio de secado fue de $60^{\circ} \mathrm{C}$.

Molienda y tamizado: en esta etapa el grano seco se reduce hasta partículas de $0.1 \mathrm{~mm}$ de diámetro. Se realizó en el Módulo de Molienda y Tamizado de marca JARCON, y en el tamizado se utilizó el tamiz de numero 120; esto facilita el posterior amasado y mezclado para obtener fideos. La harina de tarwi obtenida según la metodología de Salcedo (2009), es una harina precocida. 
Proteína: la determinación de la proteína total se realizó según el método por la N.T.P. 205.005:1979: cereales y menestras. Determinación de proteínas totales (Método de Kjeldahl), utilizando 6.25 como factor para la mayoría de los cereales y 5.70 como factor para el trigo.

Grasa: se utilizó el equipo Soxhlet, usando hexano como solvente. Metodología de la Asociación Oficial de Químicos Analistas (AOAC) 963.15 2005, método Soxhlet.

Ceniza: se realizó por la incineración de la materia orgánica en una mufla; siguiendo la metodología por la NTP 205.038:1975: harinas. Determinación de cenizas.

Fibra: se determinó después de una hidrólisis acida y luego hidrólisis alcalina; si- guiendo la metodología por la AOAC (930 $-10)$.

Carbohidratos: se obtuvo por diferencia, restando del 100\% la suma de los porcentajes de humedad $(H)$, ceniza $(C)$, grasa $(G)$, proteínas $(P)$, y fibra (F). Metodología para carbohidratos, por diferencia de materia seca (MS-INN) señalada por Collazos, et. al. (1993). Usando la fórmula: \% Carbohidratos $=100(\mathrm{H}+\mathrm{C}+-$ $\mathrm{G}+\mathrm{P}+\mathrm{F})$.

\section{RESULTADOSY DISCUSIÓN Composición químico proximal de la ha- rina de quinua}

En la tabla 1, se muestran los resultados con tres repeticiones del análisis de la composición porcentual de la harina de quinua (Chenopodium quinoa Willd).

Tabla 1. Composición porcentual (\%) de la harina de quinua.

\begin{tabular}{cc}
\hline Componentes (\%) & Quinua \\
\hline Humedad & $10.48 \pm 0.12$ \\
Proteína & 9.05 \\
Cenizas & $2.44 \pm 0.05$ \\
Grasa & $2.53 \pm 0.02$ \\
Carbohidratos & 72.39 \\
Fibra & $3.11 \pm 0.03$ \\
\hline
\end{tabular}

Los resultados mostrados en la tabla 1, nos indican que la harina de quinua tiene un porcentaje de $10.48 \pm 0.12 \%$ de humedad, los cuales se encuentran dentro de los límites máximos permisibles por la Norma Técnica Peruana 011.451:2013, ya que la humedad no debe exceder del 15\%; en las tablas peruanas de composición de alimentos-MINSA (2017) la humedad es de 11.7 \% y Dussán-Sarria et al., (2019) en su estudio determinó que la humedad es de $9.00 \pm 021 \%$; esta diferencia significativa podría deberse a las variedades de la especie vegetal y a las diferencias de lugares (Valenzuela, 2019). Las tablas peruanas de composición de alimentos-MINSA (2017), reportan un valor $12.4 \%$ de proteínas para la harina de quinua y Sotelo et al., (2019) reporta un $9.45 \%$ de proteína total, en la investigación se determinó un valor de $9.05 \%$ de proteína, valor próximo al reportado en la bibliografía de Sotelo. La harina de quinua contiene $2.44 \pm 0.05 \%$ de cenizas, valor muy cercano a lo indicado en las tablas peruanas de composición de alimentos-MINSA (2017), que reportan un valor de $2.8 \%$ de cenizas. El valor de cenizas encontrado en la investigación, se encuentra dentro de los Límites Máximos Permisibles por la Norma Técnica Peruana 011.451:2013. El contenido de grasa obtenido en la harina de quinua fue $2.53 \pm 0.02 \%$, la 
cual se encuentra dentro del rango de 2.05\% a $10.88 \%$ reportado por Rojas et al., (2016), quienes indican que el contenido de grasa de la quinua tiene un alto valor debido a su alto porcentaje de ácidos grasos no-saturados. Las tablas peruanas de composición de alimentos-MINSA (2017), reportan un valor de $67.2 \%$ de carbohidratos para la harina de quinua, que es un valor cercano al encontrado en la investigación, donde se reporta $72.39 \%$ de carbohidratos. La harina de quinua contiene $3.11 \pm 0.03 \%$ de fibra y los valores reportados por Rojas et al., (2016), son de 3,46 a 9,68\%; lo cual demuestra que nuestros datos reportados se encuentran dentro del rango a los valores reportados por la bibliografía.

\section{Composición químico proximal de la ha- rina de tarwi precocida}

En la tabla 2, se muestran los resultados con tres repeticiones del análisis de la composición porcentual de la harina de tarwi (Lupinus mutabilis Sweet).

Tabla 2. Composición porcentual (\%) de la harina de tarwi precocida.

\begin{tabular}{cc}
\hline Componentes (\%) & Tarwi \\
\hline Humedad & $6.33 \pm 0.15$ \\
Proteína & 49.04 \\
Cenizas & $2.85 \pm 0.04$ \\
Grasa & $27.32 \pm 0.25$ \\
Carbohidratos & 11.52 \\
Fibra & $2.94 \pm 0.05$ \\
\hline
\end{tabular}

En la investigación se determinó que la humedad de la harina de tarwi es de 6.33 $\pm 0.15 \%$, este valor cumple con la N.T.P. 205.044:1976, que específica que para harinas sucedáneas procedentes de leguminosas la humedad no debe exceder de 15\%. Las tablas peruanas de composición de alimentos-MINSA (2017), reportan para la harina de tarwi un valor de 7\% de humedad y Vegas \& Vegas, (2017) determinan $8,92 \pm 0,06 \%$ de humedad, valores muy cercanos obtenidos en la investigación. En la investigación se determinó un valor de 49.04\% de proteína y Vegas \& Vegas, (2017), reporta un valor de 48,06 \pm 0,34\% de proteínas, también afirma que la proteína del tarwi contiene lisina, treonina y triptófano. La harina de tarwi contiene $2.85 \pm 0.04 \%$ de cenizas, valores cercanos a lo encontrado en la bibliografía de las tablas peruanas de composición de alimentos-MINSA (2017), que reportan un valor 2.6\% de cenizas y Vegas \& Vegas, (2017), determina un valor de 2,68 $\pm 0,13 \%$ de cenizas en su investigación; el valor de cenizas determinado en el trabajo de la investigación, cumple lo establecido en la N.T.P. 205.044:1976, que específica que para harinas sucedáneas procedentes de leguminosas las cenizas no deben exceder de $5 \%$ de ceniza. La harina de tarwi, contiene $27.32 \pm 0.25 \%$ de grasa, lo reportado por Vegas $\&$ Vegas, (2017) es de 22,31 $\pm 1,20 \%$ de grasa y por las tablas peruanas de composición de alimentos-MINSA (2017), es de $27.9 \%$ de grasa, donde se comprueba que los valores de la bibliografía son cercanos a los valores obtenidos experimentalmente.

En las tablas peruanas de composición de alimentos-MINSA (2017), el valor de los carbohidratos es de $12 \%$, que es un valor cercano al encontrado en la investigación, donde se reporta 
11.52\% de carbohidratos; durante el proceso de desamargado, el grano de tarwi pierde parte de sus carbohidratos solubles, es por eso que el valor reportado es menor que la bibliografía.

La harina de tarwi contiene $2.94 \pm 0.05 \%$ de fibra y el valor reportado por Tapia et al. (2000), es de $3 \%$ de fibra, lo cual demuestra que nuestros datos reportados son similares a los reportados por la bibliografía.

\section{CONCLUSIONES}

Se determinó que la caracterización de la harina de quinua (Chenopodium quinoa Willd.) es: Proteína (9.05\%), Humedad (10.48 $\pm 0.12 \%)$, Cenizas (2.44 $\pm 0.05 \%)$, Grasa (2.53 $\pm 0.02 \%)$, Fibra (3.11 $\pm 0.03 \%)$ y Carbohidratos (72.39\%) y la caracterización de la harina de tarwi (Lupinus mutabilis Sweet) es: Proteína (49.04\%), Humedad (6.33 $\pm 0.15 \%)$, Cenizas (2.85 $\pm 0.04 \%)$, Grasa $(27.32 \pm 0.25 \%)$, Fibra $(2.94 \pm 0.05 \%)$ y Carbohidratos (11.52\%). El elevado valor nutricional que se encontró en la harina de quinua y en la harina de tarwi, demuestra que es una buena opción para empezar a realizar su industrialización en diversos productos.

\section{REFERENCIAS BIBLIOGRÁFICAS}

Calla Joel. \& Cortez Gladys. (2011). Guia técnica. Curso-taller. "Post Cosecha y Transformación de quinua orgánica". "Jornada de capacitación UNALM-AGROBANCO". Ayaviri-Puno-Perú.

Collazos, C.; Phlip, W.; Viñas, E.; Alvistur, J.; Urquieta, A.; Vásquez, J. (1993). Composición de Alimentos de mayor consumo en el Perú. 6ta Edición. Ministerio de Salud, Instituto Nacional de Nutrición. Lima, Perú.

Dussán-Sarria, S., Cruz-Noguera, R. E., \& Godoy, S. P. (2019). Estudio del Perfil de Aminoácidos y Análisis Proximal de Pastas Secas Extruidas a Base de Harina de Quinua y Harina de Chontaduro. Información tecnológica, 30(6), 93-100.
Granados, J. G. S., Tello, M. P. A., Gutierrez, A. L. L., Díaz, L. J., \& Arce, V. V. (2019). Empleo de tres métodos de desamargado a través de la evaluación sensorial de harina y pan de Lupinus mutabilis Sweet. Agroindustrial Science, 9(1), 53-59.

Instituto Nacional de Estadística e Informática, (2018). Disponible en: https://www.inei. gob.pe/prensa/noticias/desnutricion-cronica-afecto-al-122-de-la-poblacion-menor-decinco-anos-de-edad-en-el-ano-2018-11370/.

Miranda, J. P. H. (2019). La quinua como alternativa a la proteína animal en la desnutrición infantil. Revista UNIANDES de Ciencias de la Salud, 2(2), 072-081.

Murphy, K. y J. Matanguihan, (2015). Quinoa: Improvement and Sustainable Production, John Wiley \& Sons. Inc., doi:10.1002/9781118628041, 235pp, New Jersey, Estados Unidos.

Navia-Coarite, N.; Nina-Mollisaca, G.; Mena-Gallardo, E. y Salcedo-Ortiz, L.; (2019). Hidrólisis enzimática en harina de quinua y tarwi por efecto de a-amilasa. Vol. 17 No 1. Enero - Junio 2019 · ISSN - 1692-3561 - ISSN-e 1909-9959 - D Ol: http://dx.doi.org/10.18684/ bsaa.v17n1.1177.

Rojas, W., Vargas Mena, A., \& Pinto Porcel, M. (2016). La diversidad genética de la quinua: potenciales usos en el mejoramiento y agroindustria. Revista de Investigación e Innovación Agropecuaria y de Recursos Naturales, 3(2), 114-124.

Salcedo Aguirre L., (2009). Obtención de harina de Tarwi. Tesis. Universidad Mayor de San Andrés. La Paz, Bolivia.

Sotelo Méndez, A., Bernuy-Osorio, N., Vilcanqui Perez, F., Paitan Anticona, E., Ureña, M., \& Vílchez-Perales, C. (2019). Galleta elaborada 
con harina de quinua, fibras del endospermo de tara y hojas de agave: Valor biológico y aceptabilidad global. Scientia Agropecuaria, 10(1), 73-78.

Tablas peruanas de composición de alimentos-MINSA (2017). Disponible en: https:// repositorio.ins.gob.pe/xmlui/bitstream/ handle/INS/1034/tablas-peruanas-QR.pdf?sequence $=3$ \&isAllowed $=y$.

Tapia, Mario E. (2000). Cultivos andinos subexplotados y su aporte a la alimentación. (2da. Edición.). Santiago, Chile: Organización de las Naciones Unidas para la Agricultura y la Alimentación (FAO).

Valenzuela, C. (2019). Obtención de extractos proteicos por el punto isoeléctrico y composición de aminoácidos de dos variedades de Chenopodium quinoa Willd, cica 17 y cica 18. Tesis para optar al grado académico de maestro en ciencia y tecnología de alimentos. Universidad Nacional de San Antonio Abad del Cusco. Perú.

Vegas, R., Nogueroles, A. Z., \& Vegas, C. (2017). Efecto del pH y cloruro de sodio sobre las propiedades funcionales de harina de semillas de Lupinus mutabilis "tarwi" variedad criolla. Agroindustrial Science, 7(1), 49-55.

\section{CORRESPONDENCIA:}

Mag. Lucia Pantoja Tirado

luciapantoja@unat.edu.pe 